\title{
Análise do impacto do espectro do sinal de EEG na abordagem via Geometria Riemanniana
}

\author{
$1^{\text {st }}$ Demison Rolins de Souza Alves \\ Campus Universitário de Tucuruí \\ Universidade Federal do Pará \\ Belém, Brasil \\ demisonalves9@gmail.com
}

\author{
$2^{\text {nd }}$ Otávio Noura Teixeira \\ Campus Universitário de Tucuruí \\ Universidade Federal do Pará \\ Tucuruí, Brasil \\ onoura@gmail.com
}

\author{
$3^{\text {rd }}$ Cleison Daniel Silva \\ Campus Universitário de Tucuruí \\ Universidade Federal do Pará \\ Tucuruí, Brasil \\ cleison@ufpa.br
}

\begin{abstract}
Neste trabalho investiga-se o uso de uma função de pertinência Gaussiana como uma técnica para aprimorar as etapas de extração de caracteristicas e classificação em sistemas interface-cérebro maquina (ICM) baseados em imagética motora (IM). A ideia principal desta abordagem consiste em filtrar as informações espectrais do sinal do eletroencefalograma (EEG) via matrizes de covariância parametrizadas para destacar características que colaboram para classificação dos sinais através de um classificador baseado na distância de Riemann. Os resultados, em relação a performance de acurácia, adquiridos neste trabalho são validados a partir do conjunto 2 a de dados da IV competição internacional de ICM. Os resultados obtidos sugerem que a filtragem espectral realizada na abordagem via Geometria de Riemann pode afetar positivamente o desempenho do sistema ICM, aumentando sua flexibilidade.
\end{abstract}

Index Terms-Interface cérebro-máquina, Riemann, Matrizes de covariância

\section{INTRODUÇÃo}

Sistemas de interface cérebro-máquina (ICM) proporcionam uma conexão entre o cérebro e o ambiente exterior, sem o uso das vias naturais do corpo humano, fornecendo desta forma uma alternativa para o controle de dispositivos externos. Atualmente, existe uma variedade de sistemas ICM aplicados para resolução de diversas tarefas práticas [1], todavia, primeiramente, eles foram propostos como uma alternativa à outras técnicas a fim de auxiliar na reabilitação de indivíduos com capacidades motoras debilitadas [2].

A Imagética Motora (IM) é um padrão especial presente no eletroencefalograma (EEG), e tem sido utilizada em sistemas ICM. O termo faz referência à atividades cognitivas pouco complexas, como por exemplo: a imaginação motora das mãos, dos pés e mesmo, da língua [1] [2] [3].

A tarefa dos sistemas ICM requer a utilização de tecnicas robustas, pois à atividade cerebral capturada apresenta artefatos e ruidos [4] por consequência o desempenho do sistema é deteriorado. Para resolução destes problemas com frequência são aplicadas tecnicas de preprocessamento como filtros temporais e espaciais [1].

Embora este campo continue sendo amplamente explorado como podemos ver em [5] [6] [7] [8], um grande problema intrínseco persiste: a atividade cerebral difere de pessoa para pessoa. Portanto, a fim de aumentar o desempenho de qualquer sistema ICM, devemos enfatizar sua flexibilidade.
Atualmente, os classificadores baseados na Geometria Riemanninana despontam como uma alternativa proeminente para construção de sistemas BCI robustos [8], uma vez que Riemann dispõe de um framework rico para manipular os dados extraidos provenientes do sinal de EEG [9].

Neste trabalho, exploraremos a metodologia proposta por [10], que adota uma nova estratégia de extração de caracteristicas via matrizes de convariância parametrizadas, a fim de minimizar o impacto da ausência de parametros livres do classificador denominado de mínima distância para média de Riemann (RMDM), aumentando desta forma a flexibilidade do sistema ICM.

As matrizes parametrizadas têm como objetivo destacar características no domínio da frequência do sinal relevantes que possam otimizar o funcionamento do sistema. Em [10] [11] [12] essa parametrização é decretada através de um problema de otimização, entretanto, neste trabalho iremos explorar diversas sub-bandas de frequências construídas por uma função de pertinência gaussiana, desta forma podemos visualizar com maior riqueza de detalhes como a filtragem espectral do sinal de EEG interfere na perfomance do sistema ICM para cada sujeito contido no Conjunto 2a de Dados da IV Competição Internacional de ICM.

Este trabalho está estruturado da seguinte forma. $\mathrm{Na}$ sessão II, apresentamos a transformação do sinal EEG e a parametrização das matrizes de covariância. A classificação pela distância de Riemann é discutida na seção III. Na sessão IV, os resultados do experimento são comentados. Finalmente, nossas conclusões são apresentadas.

\section{EXtração DE CARACTERÍSTICAS}

\section{A. Decomposição do sinal usando uma Base Senoidal}

Em geral, o primeiro estágio para o funcionamento de um sistema ICM aplica uma filtragem ao sinal bruto de EEG. Nesta abordagem é proposta uma alternativa a filtragens com os filtros temporais convencionais. Para isso, ocorre uma projeção em uma base de sinais ortogonais formados por senoides. Essa abordagem é interessante porque emula a filtragem passa-banda e também expressa uma representação drasticamente compacta dos sinais de EEG por meio de seu conteúdo de frequêncial [12]. 
Dada a aquisição de sinais EEG, podemos descrevê-los através de uma representação vetorial que pode ser representada como:

$$
\boldsymbol{z}(n)=\left[z_{1}(n), z_{2}(n), \ldots, z_{p}(n)\right]^{T}
$$

esta definição descreve o sinal bruto coletado, em que $p$ representa o número de sensores e $\boldsymbol{n}$ um tempo discreto. Também podemos representá-lo através de um segmento de sinal matricial $Z \in \mathbb{R}^{p \times q}$, onde $\mathrm{q}$ descreve o número de amostras registradas a partir de uma taxa de amostragem $f_{s}$ [10].

A partir de $Z$ é gerada a matriz $\Gamma$ que representa os sinais filtrados e compactados no dominio da frequência. Inicialmente, devemos definir uma faixa de frequência de interesse fornecida por um limite superior $f_{u}$ e um limite inferior $f_{l}$ em hertz. Este intervalo representa à variação de frequência usada para delimitar as frequências do sinal bruto.

O sinal é projetado através da base definida pela matriz $\mathbb{B}^{2 m \times q}$ que contém $m$ sinais senoidais e cossenoidais, esta é definida por:

$b(n)=\left[\begin{array}{c}b_{1}(n) \\ \cdot \\ \cdot \\ \cdot \\ b_{2 m}(n)\end{array}\right],\left\{\begin{array}{l}b_{i}(n)=\sin \left(\frac{2 \pi f_{i}}{f_{s}} n\right) \text { para } \mathrm{i}=1, \ldots, \mathrm{m} \\ b_{m+i}(n)=\cos \left(\frac{2 \pi f_{i}}{f_{s}} n\right) \text { para } \mathrm{i}=1, \ldots, \mathrm{m}\end{array}\right.$

onde $n \in\{1, \ldots, q\}$ definindo o tempo discreto; $f_{k}$ pertence ao conjunto de frequências de interesse $\mathbf{F}=\left\{f_{1}, \ldots, f_{k}, \ldots, f_{m}\right\}$, tal que $f_{l} \geq 0 ; f_{u} \leq \frac{f_{s}}{2} ; f_{l} \leq f_{k} \leq f_{u}$ e por fim $\delta f=\frac{f_{s}}{q}$ [10] [11] [12].

A matriz $\Gamma \in \mathbb{R}^{p \times 2 m}$ é interpretada como uma matriz de coeficientes, onde $p$ está relacionado aos sensores do sinal bruto $Z$ e $\boldsymbol{m}$ aos sinais da base $B$ com frequências distintas. Esta é definida por intermedio de um problema de minimização:

$$
\begin{gathered}
\Gamma^{*}=\underset{\Gamma}{\operatorname{argmin}}\|Z-\Gamma B\|_{F}^{2} \\
\Gamma^{*}=Z B^{T}\left(B B^{T}\right)^{-1}
\end{gathered}
$$

onde $\Gamma^{*}$ significa a aproximação ótima do sinal bruto $\mathrm{Z}$ sem a perda minima de informação significante extraindo o conteudo espectral de interesse [10].

A matriz $\Gamma$ representa o sinal no domínio da frequência, no entanto, se for conveniente, podemos retornar ao domínio do tempo $X \in \mathbb{R}^{p \times q}$, através de:

$$
X=\Gamma B
$$

considerando que $X$ equivale a uma amostra do $\operatorname{sinal} Z$ filtrado a partir um filtro passa-banda dentro do intervalo de frequência de interesse [10] [12].

\section{B. Matrizes de Covariância Parametrizadas}

$\mathrm{O}$ uso de matrizes de covariância é uma estratégia comum para a construção de sistemas ICM devido à sua importância para os algoritmos de extração de características e classificação. Ela tem propriedades intrínsecamente interessantes espacialmente para os classificadores Riemannianos [9] [12], como:

- Conteúdo espacial intrínseco dos sinais de EEG

- Contidas no espaço não-euclidiano possuindo a forma de um hipercone

- Por natureza, são matrizes simétricas positivas definidas

Geralmente, ela é definida a partir da representação das amostras do sinal $X$, por:

$$
\operatorname{Cov}\{X\}=\frac{1}{q} X X^{T}
$$

assumindo que $q \gg p$. Contudo, afim de parametriza-la ela devemos considerar a seguinte transformação linear:

$$
Y=\Gamma B G=X G
$$

sendo assim pode-se determinar a matriz de covarância de $Y$ :

$$
\operatorname{Cov}\{Y\}=\frac{1}{q} \Gamma B G G^{T} B^{T} \Gamma^{T}=\Gamma H_{0} \Gamma^{T}
$$

onde aplicamos uma substituição de variável,

$$
H_{0} \triangleq \frac{1}{q} B G G^{T} B^{T} \in \mathbb{R}^{2 m \times 2 m}, H_{0} \succeq 0
$$

Dada a matriz $H_{0}$, a transformação $G$ pode ser definida por $G=B^{T}\left(B B^{T}\right)^{-} 1\left(q H_{0}\right)^{\frac{1}{2}}$, contudo, em termos de otimização de classificação determinar $H_{0}$ é suficiente [10].

A matriz $H_{0} \in \mathbb{R}^{2 m \times 2 m}$ tem uma restrição intrínseca para ser positiva semi-definida. É utilizada para alterar propriedades da matriz de covariância. Como podemos ver na Eq (8), a matriz de covariância é construída usando $\Gamma$, desta forma $H_{0}$ tem o potencial para ponderar suas colunas e consequemente a informação frequencial do sinal.

Definida a matriz $H_{0}$, o problema agora é como determinar seus valores para destacar as informações de frequência mais relevantes para aumentar a taxa de classificação.

\section{Usando uma função gaussiana para determinar $H_{0}$}

A função de pertinência gaussiana é popularmente apresentada principalmente em sistemas fuzzy, porém, neste trabalho ela será aplicada para construção da matriz $H_{0}$, justamente por ser simples construí-la a partir de apenas dois parâmetros, média e desvio padrão.

Por natureza a matriz $H_{0} \in \mathbb{R}^{2 m \times 2 m}$ logo em teoria ela possui $2 \mathrm{~m}(2 \mathrm{~m}+1) / 2$ parâmetros a serem determinados, contudo, em [10] [12] ela é descrita como uma matriz diagonal ou pentadiagonal. Neste trabalho adotaremos uma matriz diagonal e ainda reduzermos pela metade o número de parametros, pois a matriz $\Gamma$ possui duas colunas relacionadas à mesma faixa de frequência, desta forma decidimos usar apenas m parâmetros e aplicá-los ás colunas correspondentes. 
Os elementos da matriz $H_{0}$ são definidos a partir da função de pertinência gaussiana:

$$
h(x, c, \sigma)=e^{\frac{-(x-c)^{2}}{2 \sigma^{2}}}
$$

onde $x$ é frequência que desejamos ponderar; $c$ é a média e $\sigma$ o desvio padrão. Neste trabalho os valores possiveis para média estão contindos no conjunto de frêquencia de interesse $\mathbf{F}$ descrito em II-A.

\section{ClassificaÇÃo USAndo A DistÂnCIA DE RIEMANN}

Métricas de distância são comumente usadas em algoritmos de classificação. Atualmente, a estrutura de geometria de Riemann oferece uma excelente alternativa para a construção de sistemas ICM. Isso se deve à importância das matrizes de covariância, conforme visto na seção anterior. Os classificadores Riemannianos despontaram como ótimas ferramentas, pois uma métrica de distância não euclidiana se encaixa perfeitamente neste contexto.

\section{A. Mínima Distância para Média de Riemann}

Proposto por [9], o princípio desta técnica baseia-se no uso de matrizes de covariância como informação direta do sinal EEG, juntamente com a geometria de Riemann para classificar os sinais, pois eles possuem uma estrutura específica que pertence à variedade Riemanniana de matrizes simétricas positivas definida; portanto, a geometria de Riemann possui uma estrutura robusta tornando-se assim uma excelente ferramenta para manipular as matrizes de covariância. [9] [13]

A métrica de distância de Riemann é definida pelo tamanho geodesica que é da curva minima que conecta dois ponto na Varieade de Riemann, podemos defini-la através de:

$$
\delta_{R}\left(P_{1}, P_{2}\right)=\left\|\log \left(P_{1}^{-1} P_{2}\right)\right\|_{F}=\left[\sum_{i=1}^{q} \log ^{2} \lambda_{i}\right]^{\frac{1}{2}}
$$

onde $P_{1}$ e $P_{2}$ são dois pontos quaisquer e \|\|$_{F}$ significa a norma de frobenius; $\lambda_{i}$ descreve os autovalores da matriz $P_{1}^{-1} P_{2}$ [9].

É interesante destacar como a construção da Eq (11) é definida. Dada dois pontos $P_{1}$ e $P_{2}$ (duas matrizes) não há alternativa para manipula-las através do espaço via transformações espaciais, pois por natureza a distância de Riemann é invariante à transformações de similaridade e de congruência [9].

Isso torna a abordagem via distância de Riemann um algoritmo livre de parametros, uma vez que o sinais de EEG já estejam filtrados em um intervalo de frequência de interesse. Por esse motivo, a abordagem vista em [10] [11] [12] propondo a introdução de parâmetros livres via matrizes de covariâncias parametrizadas no dominio da frequência é interessante, pois há capacidade de flexibilizar à abordagem via Riemann canônica.

A partir da descrição da métrica podemos apresentar o funcionamento do RMDM. Inicialmente para realizar a classificação dos sinais, o primeiro passo é calcular a média geométrica para as matrizes de covariância [9] [10] via:

$$
M_{g} *=\underset{M_{g}}{\operatorname{argmin}} \sum_{i=1}^{k} \delta_{R}^{2}\left(M_{g}, \operatorname{Cov}\{Y\}_{i}\right)
$$

Para cada classe devemos obter uma matriz média correspondente, por exemplo, supondo que existam duas classes 1 e 2 que queremos idenficar, teremos as matrizes médias $M_{g 1}$ e $M_{g 2}$. Para definir se um seguimento do sinal $\Omega$ pertece à classe 1 ou 2 apenas calcule a distância de Riemann via Eq (11), entre as médias e $\Omega$ caso ele esteja mais proximo de $M_{g 1}$ então $\Omega \in 1$ caso contrario $\Omega \in 2$.

\section{EXPERIMENTO}

\section{A. Conjunto de Dados}

O conjunto de dados foi utilizado através do repositório público da competição internacional de número IV de sistemas ICM; o conjunto de dados 2 a apresenta sinais de EEG de nove sujeitos, para cada qual realizou quatro tarefas cognitivas distintas: imaginação do movimento da mão esquerda (classe 1); mão direita (classe 2); pés (classe 3) e língua (classe 4). O número de eletrodos de EEG posicionados foi igual a 22; descritos por uma taxa de amostragem de $250 \mathrm{~Hz}$. Neste conjunto de dados, há uma divisão própia entre o conjunto de teste e treinamento, para o qual cada um possui 72 testes para cada tarefa motora imaginária descrita anteriormente, o restante dos dados existentes, que são três sensores de eletrooculografia, não serão utilizados neste trabalho [14].

\section{B. Pre-processamento}

A janela extraída inicia aos 2,5s até os 4,5s; 500 amostras por seguimento; $f_{l}=8 \mathrm{~Hz}$ e $f_{u}=30 \mathrm{~Hz}$; espaçamento entre as frequências $\delta f=0.5 ; \mathrm{m}=45$ frequencias distintas seguindo as diretrizes dos experimentos apresentados em [11] e [12]. Para construção da função gaussiana a média é definida pelo conjunto $\mathcal{F}$ de frequências $\{8,8.5,9, \ldots, 29.5,30\}$ e o desvio padrão é definido empiricamente entre o conjunto $\{2,3,4,5\}$, pois, valores definidos inferiores a 2 podem ocasionar problemas númericos ao manipular as matrizes de covariância via Riemann.

\section{Classificação}

Os resultados nesta seção são provenientes da própria divisão, conjunto de treino e teste, oferecida pelo repositório público $2 \mathrm{a}$ da competição internacional de número IV de sistemas ICM. Os resultados apresentados nesta seção serão apenas relacionados as tarefas de IM da mão esquerda e direita de cada sujeito.

$\mathrm{Na}$ abordagem de Distância de Riemann, não há necessidade de treinamento convencional. O conjunto de treinamento é utilizado para construir pontos de referência no espaço, definindo a média geométrica correspondente de cada classe a ser identificada como podemos ver na Eq (12). 


\section{Implementação}

Para comparação de desempenho, duas abordagens foram aplicadas nesta sessão: classificador usando a distância de Riemann onde a matriz $H_{0}=I$ (isto significa $H_{0}$ igual um matriz identidade, logo a informação frequêncial permanesse inalterada) e matriz $H_{0}=D$ definido pela função de pertinência gaussiana diagonal.

\section{E. Resultados e Discussões}

Nas Figuras 1 e 2, podemos ver no eixo Y do gráfico como a construção da matriz $H_{0}$ impacta a precisão do sistema. No eixo $\mathrm{X}$, vemos as 45 frequências distintas $\in \mathrm{F}$ utilizadas como média para a função de pertinência gaussiana; 4 configurações de desvio padrão, totalizando 180 configurações $H_{0}=D$ para cada sujeito.

Antes de iniciar a comparação $H=I$ vs $H=D$, é interessante destacar a suavidade na diferença de precisão quando usamos a frequência vizinha para construir $H_{0}$ e à medida que o desvio padrão aumenta, torna-se mais evidente. Em geral, há uma diminuição ou aumento relativamente pequeno na precisão a medida que deslocamos o centro da gaussiana para frequências vizinhas.

Vale destacar principalmente para o sujeito 1 , onde é bastante evidente que mesmo com a curva gaussiana mais estreita (desvio padrão $=2$ ) existem diferentes frequências relevantes, por exemplo, quando o centro da gaussiana assume valores entre $10-12 \mathrm{~Hz}$ e $24-26 \mathrm{~Hz}$. Como tal, isso pode sugerir combiná-las seja ser uma estratégia em potencial.

Mesmo mencionado anteriormente e já conhecido na literatura, é interessante observar como a atividade cerebral difere de sujeito para sujeito e isso fica evidente quando olhamos os gráficos das Figuras 1 e 2. Em geral, podemos dizer que para cada sujeito o desempenho das matrizes $H_{0}$ é distinto.

Na Figura 3, há uma comparação entre as taxas de precisão de $H_{0}=I$ e a melhor matriz $H_{0 \text { melhor }}$ de cada sujeito. Podemos observar que para todos sujeitos $H_{0 \text { melhor }}$ apresentou um desempenho de acurácia superior ou equivalente a matriz $H_{0}=I$. Vale enfatizar crescimento considerável de acurácia obtida para os sujeitos 5 e 7 aproximadamente de $\mathbf{1 0 \%}$ e $\mathbf{2 2 \%}$.

Observando de forma geral através da média entre os nove sujeitos $H_{0 \text { melhor }}$ obteve um desempenho superior de $\mathbf{5 , 3 2 \%}$, isso sugere que o sistema ICM pode obter um ganho significativo na taxa de classificação ao filtrar o espectro do sinal de EEG via Geometria de Riemann.

\section{CONClus Ão}

Neste trabalho, a partir de uma nova representação do sinal, graus de liberdade são introduzidos ao classificador baseado na distância mínima para a média de Riemann por meio de matrizes de covariâncias parametrizadas. Essa parametrização, aprimora o sinal em alguns casos, destacando determinados intervalos de frequência, melhorando assim a etapa de extração de características e por consequência a performance geral do sistema ICM.

Esta abordagem torna o método maleável, destacando a informação espectral do sinal de EEG. Assim, há uma mudança de paradigma pela introdução de parâmetros livres determinados pela matriz $H_{0}$ removendo a invariábilidade encontrada na abordagem clássica devido as propriedades encontradas na Geometria de Riemann.

Embora as sub-bandas de frequência sejam determinadas empiricamente por tentiva e erro através da função de pertinência gaussiana, é interessante observar o quão significante para taxa de classificação é a filtragem das informações frequências para cada sujeito individual. Assim, trabalhos futuros consideram propor sua sintonia automatica via Algoritmos de aprendizado de maquina extraindo as informações das sub-bandas de frequências discutidas neste trabalho.

\section{REFERENCES}

[1] J. Wolpaw and E. W. Wolpaw, Brain-computer interfaces: principles and practice. OUP USA, 2012.

[2] J. R. Wolpaw, N. Birbaumer, D. J. McFarland, G. Pfurtscheller, and T. M. Vaughan, "Brain-computer interfaces for communication and control," Clinical neurophysiology, vol. 113, no. 6, pp. 767-791, 2002.

[3] J. Annett, "On knowing how to do things: a theory of motor imagery," Cognitive Brain Research, vol. 3, no. 2, pp. 65-69, 1996.

[4] M. Teplan et al., "Fundamentals of eeg measurement," Measurement science review, vol. 2, no. 2, pp. 1-11, 2002.

[5] F. Lotte, M. Congedo, A. Lécuyer, F. Lamarche, and B. Arnaldi, "A review of classification algorithms for eeg-based brain-computer interfaces," Journal of neural engineering, vol. 4, no. 2, p. R1, 2007.

[6] D. J. Krusienski, M. Grosse-Wentrup, F. Galán, D. Coyle, K. J. Miller, E. Forney, and C. W. Anderson, "Critical issues in state-of-the-art braincomputer interface signal processing," Journal of neural engineering, vol. 8 , no. 2 , p. $025002,2011$.

[7] L. F. Nicolas-Alonso and J. Gomez-Gil, "Brain computer interfaces, a review," sensors, vol. 12, no. 2, pp. 1211-1279, 2012.

[8] F. Lotte, L. Bougrain, A. Cichocki, M. Clerc, M. Congedo, A. Rakotomamonjy, and F. Yger, "A review of classification algorithms for eegbased brain-computer interfaces: a 10 year update," Journal of neural engineering, vol. 15, no. 3, p. 031005, 2018.

[9] A. Barachant, S. Bonnet, M. Congedo, and C. Jutten, "Multiclass brain-computer interface classification by riemannian geometry," IEEE Transactions on Biomedical Engineering, vol. 59, no. 4, pp. 920-928, 2011.

[10] C. Silva, "Processamento de sinais de eeg para classificação de tarefas motoras em sistemas de interface cérebro-máquina," Ph.D. dissertation, Universidade Federal de Santa Catarina, UFSC, 052017.

[11] C. Silva, R. Duarte, and A. Trofino, "Feature extraction improvements using an Imi approach and riemannian geometry tools: An application to bci," in 2016 IEEE Conference on Control Applications (CCA). IEEE, 2016, pp. 966-971.

[12] C. Silva, R. Duarte, R. Goulart, and A. Trofino, "Towards a lmi approach to feature extraction improvements and classification by riemann distance," in 2016 12th IEEE International Conference on Control and Automation (ICCA). IEEE, 2016, pp. 990-995.

[13] M. Congedo, A. Barachant, and R. Bhatia, "Riemannian geometry for eeg-based brain-computer interfaces; a primer and a review," BrainComputer Interfaces, vol. 4, no. 3, pp. 155-174, 2017.

[14] C. Brunner, R. Leeb, G. Müller-Putz, A. Schlögl, and G. Pfurtscheller, "Bci competition 2008-graz data set a," Institute for Knowledge Discovery (Laboratory of Brain-Computer Interfaces), Graz University of Technology, vol. 16, pp. 1-6, 2008. 

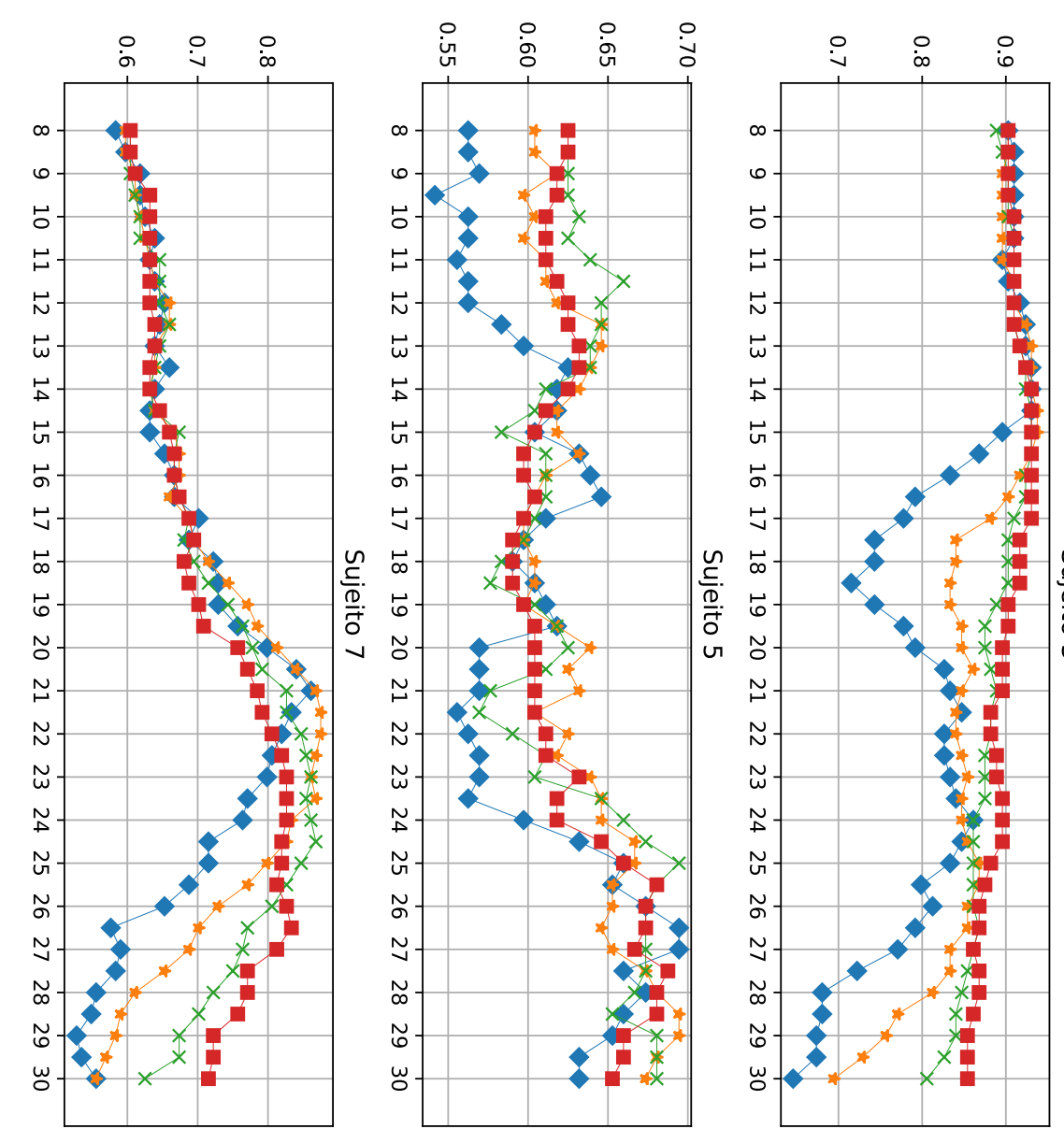

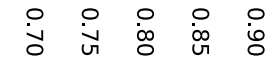
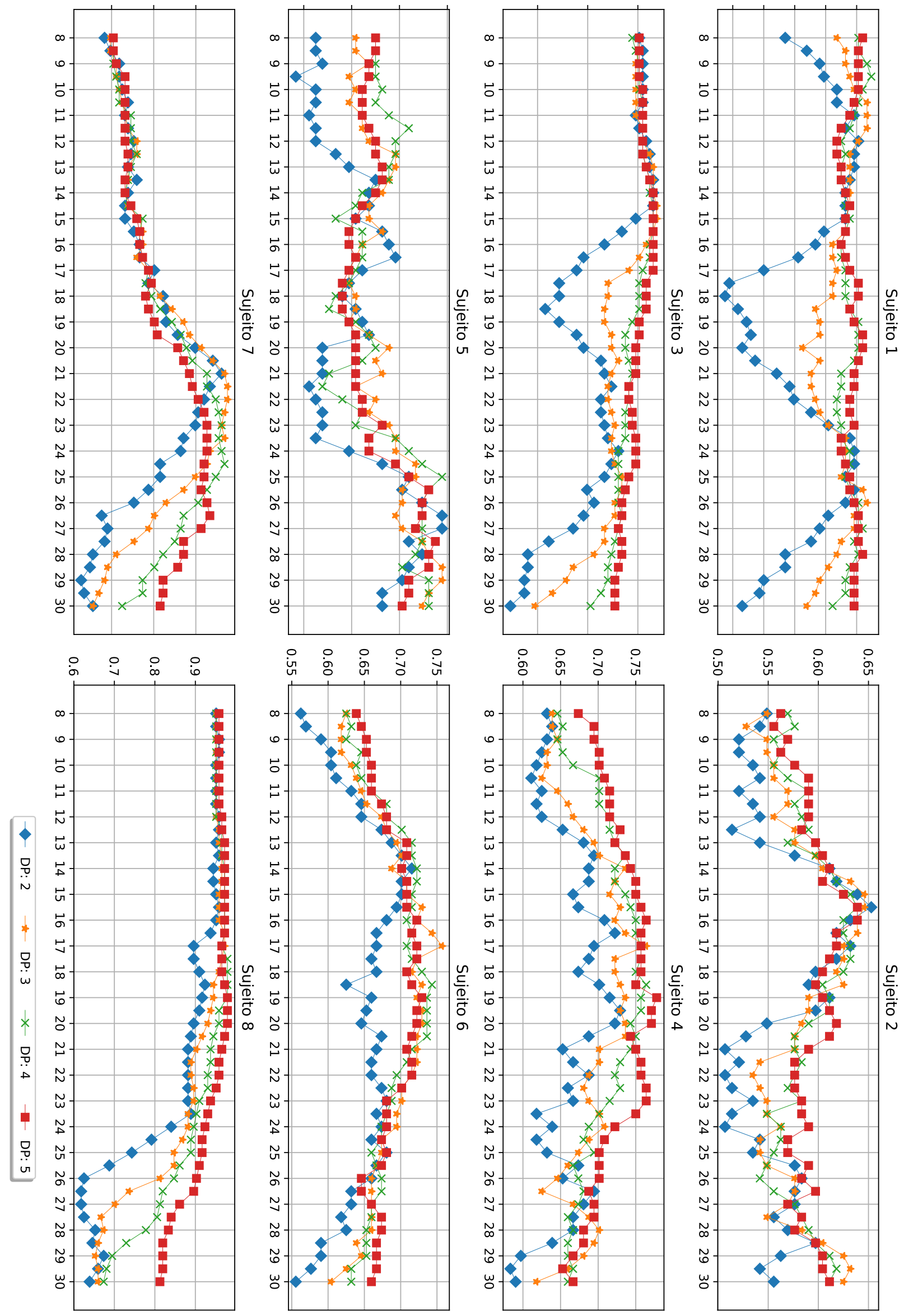

Fig. 1. Acurácia obtida para cada configuração de $H_{0}$ dos sujeitos de 1-8 
Sujeito 9

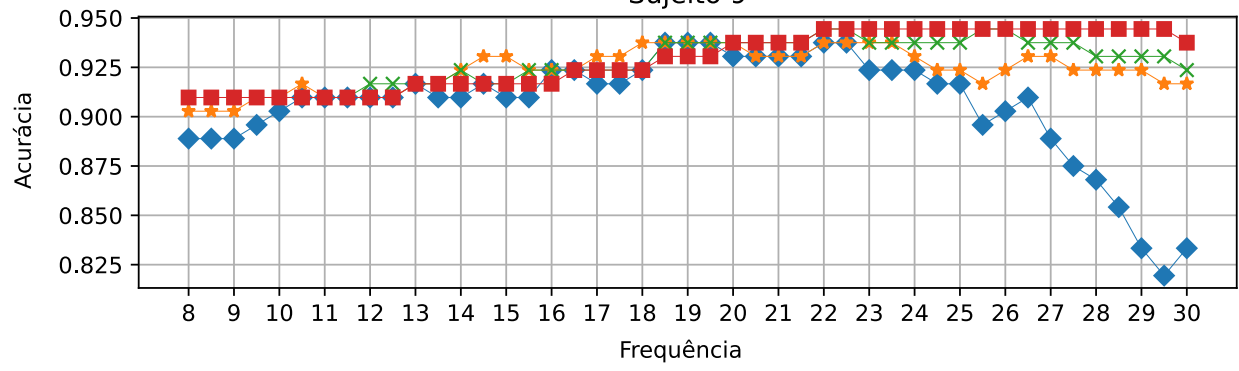

DP: $2 \rightarrow$ DP: $3 \rightarrow$ DP: $4 \div$ DP: 5

Fig. 2. Acurácia obtida para cada configuração de $H_{0}$ do sujeito 9

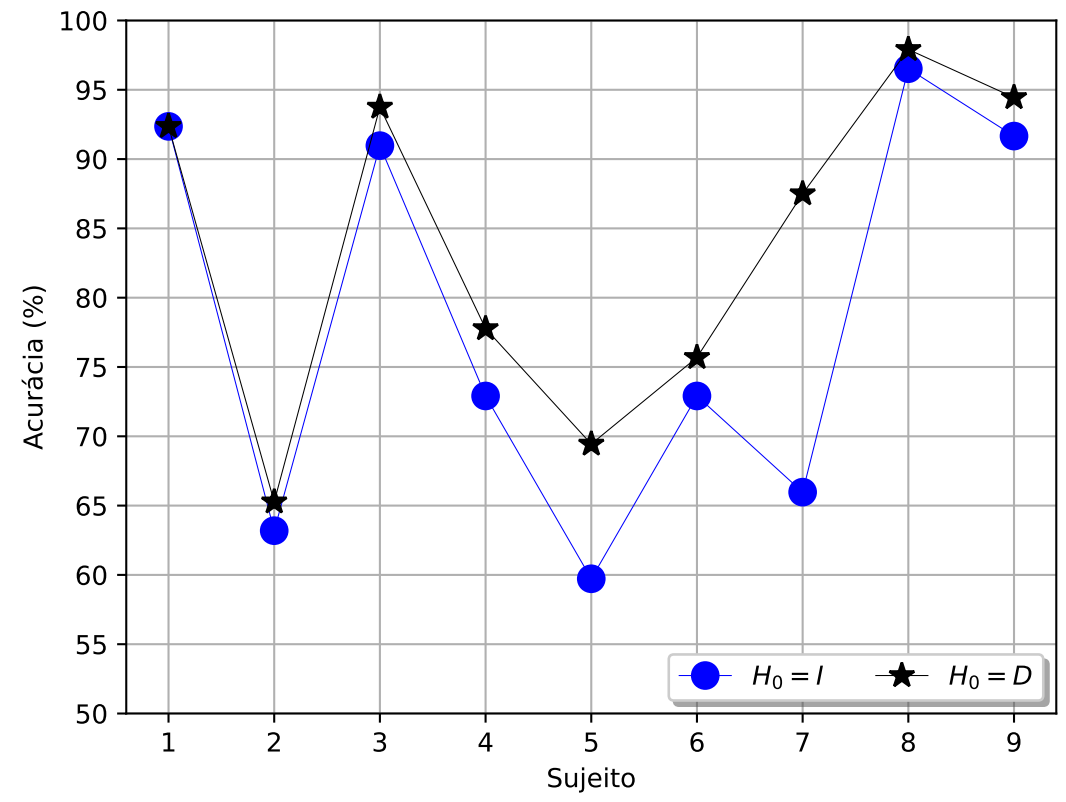

Fig. 3. Acurácia (\%) usando $H_{0}=I$ vs $H_{0 \text { melhor }}=D$ para cada sujeito 